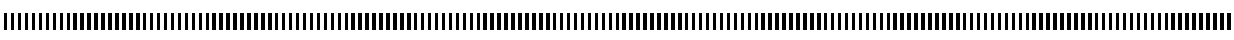

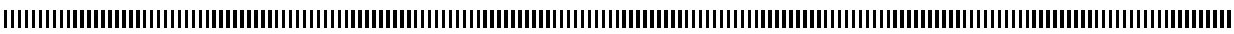

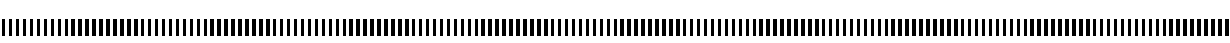

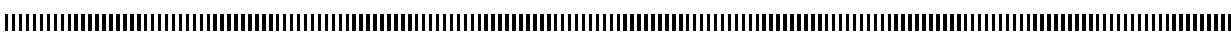

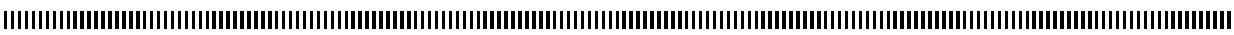

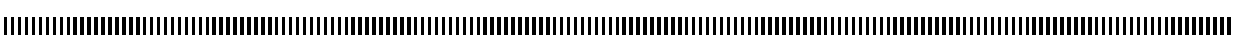

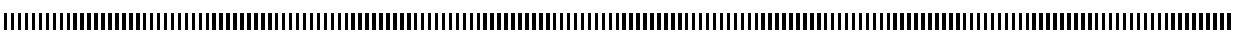

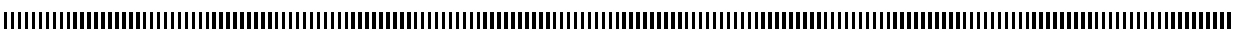

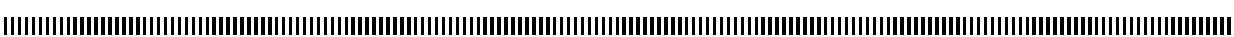

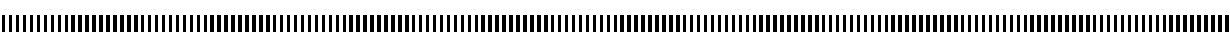

Special issue CARI'12

\title{
Analyse mathématique d'un modèle de digestion anaérobie à trois étapes
}

\author{
Radhouane Fekih-Salem ${ }^{a, e, *}$ - Nahla Abdellatif a,b — Tewfik Sari c,e_ \\ Jérôme Harmand d,e \\ a Université de Tunis El Manar, École Nationale d'Ingénieurs de Tunis, LAMSIN, \\ B.P. 37, 1002 Tunis Le Belvédère, Tunisie. (e-mail: radhouene.fs@ gmail.com) \\ b Université de Manouba, École Nationale des Sciences de l'Informatique, \\ Campus Universitaire de Manouba, 2010 Manouba, Tunisie. (e-mail: nahla.abdellatif@ensi.rnu.tn) \\ c Irstea, UMR Itap, \\ 361 rue Jean-François Breton, 34196 Montpellier Cedex, France. (e-mail: tewfik.sari@irstea.fr) \\ d Inra, UR0050, Laboratoire de Biotechnologie de l'Environnement, \\ Avenue des étangs, 11100 Narbonne, France. (e-mail: harmand@supagro.inra.fr) \\ e EPI Modemic Inra-Inria, UMR Mistea, SupAgro Bât 21, \\ 2, place Pierre Viala, 34060 Montpellier, France. \\ * Corresponding author.
}

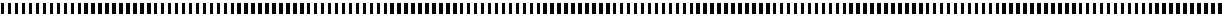

RÉSUMÉ. Dans ce travail, on s'intéresse à l'analyse mathématique du modèle d'un chémostat avec dégradation enzymatique du substrat (matière organique) qui peut se trouver sous forme solide [7]. L'étude du modèle à trois étapes est déduite d'un sous-modèle de plus faible dimension puisque certaines variables peuvent être découplées des autres. On étudie l'existence et la stabilité des points d'équilibre du sous-modèle pour des taux de croissance monotones et des taux de dilution distincts. Il est bien connu que le modèle classique du chémostat, avec des taux de croissance monotones, n'admet qu'un seul point d'équilibre globalement attractif et qu'une bistabilité ne peut pas se produire [8]. Ici, on montre que le sous-modèle étudié peut présenter une bistabilité alors que tous les taux de croissance considérés sont monotones. L'étude du modèle à trois étapes montre l'existence d'au plus quatre équilibres strictement positifs dont un est localement asymptotiquement stable. Selon la condition initiale considérée, une seule ou plusieurs espèces microbiennes ont des points d'équilibre positifs.

ABSTRACT. In this work, we focus on the mathematical analysis of a model of chemostat with enzymatic degradation of a substrate (organic matter) that can partly be under a solid form [7]. The study of this 3-step model is derived from a smaller order sub-model since some variables can be decoupled from the others. We study the existence and the stability of equilibrium points of the sub-model considering monotonic growth rates and distinct dilution rates. In the classical chemostat model with monotonic kinetics, it is well known that only one equilibrium point attracts all solutions and that bistability never occurs [8]. In the present study, although only monotonic growth rates are considered, it is shown that the considered sub-model may exhibit bistability. The study of 3-step model shows the existence at most four positive equilibrium whose one is locally asymptotically stable and according to the initial condition the two species can coexist.

MOTS-CLÉS : Bistabilité, Chémostat, Dégradation enzymatique, Hydrolyse, Taux de croissance.

KEYWORDS : Bistability, Chemostat, Enzymatic degradation, Growth rate, Hydrolysis.

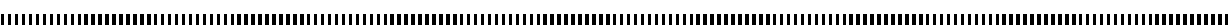




\section{Introduction}

La digestion anaérobie est un procédé biologique dans lequel la matière organique est transformée par des micro-organismes en méthane et dioxyde de carbone (biogaz) en l'absence d'oxygène. La recherche de modèles appropriés et destinés à être utilisés dans les problèmes de contrôle est aujourd'hui une priorité importante pour optimiser les processus de fermentation et résoudre des problèmes importants tels que le développement d'énergie renouvelable à partir des eaux usées et des déchets. Du point de vue microbiologique, biochimique et technologique, la digestion anaérobie est généralement considérée comme composée de trois étapes : L'hydrolyse et la liquéfaction des molécules organiques de grande taille insolubles, par des enzymes extracellulaires; une étape de production d'acides par un consortium microbien acidogène et une étape de production du méthane par un écosystème méthanogéne. Plusieurs modèles mathématiques liés à ces phénomènes ont été proposés dans la littérature. Ils sont cependant souvent très complexes et non appropriés pour le contrôle $[7,1,2,3]$. La manière la plus appropriée pour modéliser l'hydrolyse fait encore débat. En pratique, deux visions dominent : on peut supposer qu'il s'agit d'un phénomène purement enzymatique ou alors considérer le rôle majeur des bactéries hydrolytiques et donc rajouter un compartiment microbien particulier au modèle. Cette étape réactionnelle peut donc être modélisée de différentes manières :

- On peut considérer que l'activité microbienne enzymatique est constante sans faire intervenir explicitement un compartiment microbien hydrolytique. La vitesse de réaction est alors $r_{0}=k_{h y d} X_{0}$ où $k_{h y d}$ est une constante et où $X_{0}$ désigne la concentration du substrat lentement biodégradable.

- On peut diviser le compartiment du substrat en deux parties : le substrat lentement biodégradable $X_{0}$ et le substrat facilement biodégradable $S_{1}$. Pour garder le modèle assez simple, on peut supposer de plus que le même consortium bactérien $X_{1}$ dégrade ces deux substrats. La vitesse de réaction est alors $r_{0}=\mu_{0}\left(X_{0}\right) X_{1}$ où $\mu_{0}$ désigne le taux de croissance spécifique de $X_{1}$ sur $X_{0}$. Les différentes étapes réactionnelles de la digestion anaérobie sont résumés dans le tableau suivant :

\begin{tabular}{l||l}
\hline \multicolumn{1}{l||}{ Étapes } & Réactions biologiques \\
\hline \hline Hydrolyse & $X_{0} \stackrel{r_{0}}{\longrightarrow} k_{0} S_{1}$ \\
\hline \hline Acidogénèse & $k_{1} S_{1} \stackrel{r_{1}}{\longrightarrow} X_{1}+k_{2} S_{2}+C_{2}$ \\
\hline \hline Méthanogénèse & $k_{3} S_{2} \stackrel{r_{2}}{\longrightarrow} X_{2}+C_{2}+C H_{4}$ \\
\hline
\end{tabular}

où $r_{i}=\mu_{i}\left(S_{i}\right) X_{i}, i=1,2$, désignent respectivement les vitesses de réaction et $\mu_{i}, i=$ 1,2 , les taux de croissance spécifique de $X_{i}$ sur $S_{i}$ avec $S_{2}$ la concentration en intermédiaires métaboliques que sont les acides gras volatiles et $X_{2}(t)$ la concentration des bactéries méthanogénes. Finalement, $k_{i}, i=0, \ldots, 3$, désignent les coefficients de rendement associés aux réactions biologiques. 
On considère que le chémostat fonctionne en mode continu, c'est-à-dire que le débit d'entrée $Q$ est égal au débit de sortie. On note par ailleurs $D=\frac{Q}{V}$ le taux de dilution du système avec $V$ le volume du réacteur. Souhaitant étudier ce modèle dans un contexte le plus générique possible, nous considérons la possibilité de découpler le temps de séjour des solides et le temps de séjour hydraulique $\left(\frac{1}{D}\right)$ en introduisant le paramètre $\alpha, \alpha \in$ $[0,1]$, qui représente la fraction de la biomasse qui quitte le réacteur ainsi que proposé par [4] pour modéliser un réacteur à biomasse fixée. Avec ces notations, le modèle à trois étapes s'écrit :

$$
\left\{\begin{aligned}
\dot{X}_{0} & =D X_{0 i n}-\alpha D X_{0}-r_{0} \\
\dot{S}_{1} & =D\left(S_{1 i n}-S_{1}\right)+k_{0} r_{0}-k_{1} \mu_{1}\left(S_{1}\right) X_{1} \\
\dot{X}_{1} & =\left(\mu_{1}\left(S_{1}\right)-\alpha D\right) X_{1} \\
\dot{S}_{2} & =D\left(S_{2 i n}-S_{2}\right)+k_{2} \mu_{1}\left(S_{1}\right) X_{1}-k_{3} \mu_{2}\left(S_{2}\right) X_{2} \\
\dot{X}_{2} & =\left(\mu_{2}\left(S_{2}\right)-\alpha D\right) X_{2}
\end{aligned}\right.
$$

où $r_{0}=K_{h y d} X_{0}$ ou bien $r_{0}=\mu_{0}\left(X_{0}\right) X_{1}, X_{0 i n}$ désigne la concentration du substrat lentement biodégradable à l'entrée du chémostat et $S_{j i n}, j=1,2$, la concentration du substrat $j$ à l'entrée du chémostat. Avant de passer à l'analyse de ce modèle, les hypothèses suivantes sont introduites. D'après le principe de conservation de la matière, on a les inégalités :

$$
\int_{t_{1}}^{t_{2}} r_{0} V d \tau \geqslant \int_{t_{1}}^{t_{2}} k_{0} r_{0} V d \tau \Longleftrightarrow 1 \geqslant k_{0},
$$

qui s'interprètent de la manière suivante : pendant une unité de temps, la quantité de $X_{0}$ dégradée est supérieure ou égale à la quantité de $S_{1}$ produite. De même, on a $k_{1} \geqslant 1+k_{2}$ et $k_{3} \geqslant 1$, c'est-à-dire que la quantité de $S_{1}$ dégradée est supérieure ou égale à la quantité de biomasse $X_{1}$ qui s'est développée et de $S_{2}$ produit. La quantité de $S_{2}$ dégradée est supérieure ou égale à la quantité de biomasse $X_{2}$ qui s'est développée.

Cet article est organisé comme suit. Tout d'abord, on étudie le modèle (1) en supposant que le taux de croissance $\mu_{1}$ est monotone et $\mu_{2}$ est non monotone avec $r_{0}=k_{h y d} X_{0}$. Ce modèle est dit sans compartiment microbien hydrolytique. Ensuite, on étudie le sousmodèle avec compartiment microbien hydrolytique (3) en supposant que les taux de croissance $\mu_{i}, i=0,1$ sont monotones avec $r_{0}=\mu_{0}\left(X_{0}\right) X_{1}$. On montre que le sous-modèle peut présenter une bistabilité. En outre, on étudie l'existence et le comportement asymptotique des points d'équilibre du modèle à trois étapes. On démontre l'existence d'au plus neuf points d'équilibre dont quatre sont strictement positifs et dont un est Localement Asymptotiquement Stable (LAS). Finalement, on illustre les résultats mathématiques démontrés par des simulations numériques.

\section{Modèle sans compartiment microbien hydrolytique}

On suppose que

H1 : La fonction $\mu_{1}($.$) est monotone avec \mu_{1}(0)=0$. L'équation $\mu_{1}\left(S_{1}\right)=\alpha D$ admet une unique solution $\lambda_{1}=\mu_{1}^{-1}(\alpha D)$.

H2 : La fonction $\mu_{2}($.$) est non monotone avec \mu_{2}(0)=0$. L'équation $\mu_{2}\left(S_{2}\right)=\alpha D$ admet deux solutions $\lambda_{2}^{i}, i=1,2$, tel que $\lambda_{2}^{1}<\lambda_{2}^{2}$. 
Si $r_{0}=k_{h y d} X_{0}$, alors la première équation du système (1) dépend seulement de la variable $X_{0}$, et $X_{0}$ converge globalement vers son équilibre

$$
X_{0}^{*}=\frac{D}{k_{h y d}+\alpha D} X_{0 i n}
$$

À l'équilibre de $X_{0}$, les quatre dernières équations du système (1) se réduisent au modèle AM2 [2, 3] suivant

$$
\left\{\begin{array}{l}
\dot{S}_{1}=D\left(S_{1 i n}^{*}-S_{1}\right)-k_{1} \mu_{1}\left(S_{1}\right) X_{1} \\
\dot{X}_{1}=\left(\mu_{1}\left(S_{1}\right)-\alpha D\right) X_{1} \\
\dot{S}_{2}=D\left(S_{2 i n}-S_{2}\right)+k_{2} \mu_{1}\left(S_{1}\right) X_{1}-k_{3} \mu_{2}\left(S_{2}\right) X_{2} \\
\dot{X}_{2}=\left(\mu_{2}\left(S_{2}\right)-\alpha D\right) X_{2}
\end{array}\right.
$$

où

$$
S_{1 i n}^{*}=S_{1 i n}+\frac{k_{0} k_{h y d}}{k_{h y d}+\alpha D} X_{0 i n} .
$$

À chaque équilibre $F=\left(S_{1}^{*}, X_{1}^{*}, S_{2}^{*}, X_{2}^{*}\right)$ du modèle (2) correspond un équilibre $E=$ $\left(X_{0}^{*}, S_{1}^{*}, X_{1}^{*}, S_{2}^{*}, X_{2}^{*}\right)$ du modèle (1). De plus, la stabilité locale de ces derniers est déduite de celle des équilibres correspondants $F$ de (2). L'analyse du système (2) a été faite dans $[2,3]$. On obtient le résultat suivant :

Proposition 2.1 Les points d'équilibre du système (1) sont donnés par

1) $E_{0}=\left(X_{0}^{*}, S_{1 i n}^{*}, 0, S_{2 i n}, 0\right)$ qui existe toujours.

2) $E_{1}^{i}=\left(X_{0}^{*}, S_{1 i n}^{*}, 0, \lambda_{2}^{i}, X_{2}^{i}\right), i=1,2$, avec $X_{2}^{i}=\frac{1}{k_{3} \alpha}\left(S_{2 i n}-\lambda_{2}^{i}\right)$, qui existe si et seulement si $S_{2 i n}>\lambda_{2}^{i}$.

3) $E_{2}^{0}=\left(X_{0}^{*}, \lambda_{1}, X_{1}^{*}, S_{2 i n}^{*}, 0\right)$ avec

$$
X_{1}^{*}=\frac{1}{k_{1} \alpha}\left(S_{1 i n}^{*}-\lambda_{1}\right) \quad \text { et } \quad S_{2 i n}^{*}=S_{2 i n}+\frac{k_{2}}{k_{1}}\left(S_{1 i n}^{*}-\lambda_{1}\right),
$$

qui existe si et seulement si $S_{1 \text { in }}^{*}>\lambda_{1}$.

4) $E_{2}^{i}=\left(X_{0}^{*}, \lambda_{1}, X_{1}^{*}, \lambda_{2}^{i}, X_{2}^{* i}\right), i=1,2$, avec $X_{2}^{* i}=\frac{1}{k_{3} \alpha}\left(S_{2 i n}^{*}-\lambda_{2}^{i}\right)$, qui existe si et seulement si $S_{1 \text { in }}^{*}>\lambda_{1}$ et $S_{2 i n}^{*}>\lambda_{2}^{i}$.

On rappelle que la condition de persistance des espèces $X_{1}$ dans le modèle AM2 est $\lambda_{1}<S_{1 i n}$, [2, 3]. Par l'effet de l'hydrolyse, $S_{1 \text { in }}$ a été augmentée à $S_{1 i n}^{*}$. La condition de persistance de l'espèce $X_{1}$ dans le modèle d'hydrolyse devient $\lambda_{1}<S_{1 \text { in }}^{*}$; assez logiquement puisque recevant davantage de substrat, l'espèce 1 se trouve favorisée par l'ajout du terme d'hydrolyse.

\section{Modèle avec compartiment microbien hydrolytique}

Dans la suite, on s'intéresse à l'étude du sous-modèle donné par les trois premières équations du système (1) avec $r_{0}=\mu_{0}\left(X_{0}\right) X_{1}$, qu'on pourra découpler des deux dernières équations, puisque les trois premières équations ne dépendent pas des variables $X_{2}$ 
et $S_{2}$. Ainsi, on se propose d'étudier l'existence et la stabilité des points d'équilibre du sous-modèle suivant :

$$
\left\{\begin{array}{l}
\dot{X}_{0}=D\left(X_{0 i n}-\alpha X_{0}\right)-\mu_{0}\left(X_{0}\right) X_{1} \\
\dot{S}_{1}=D\left(S_{1 i n}-S_{1}\right)+k_{0} \mu_{0}\left(X_{0}\right) X_{1}-k_{1} \mu_{1}\left(S_{1}\right) X_{1} \\
\dot{X}_{1}=\left(\mu_{1}\left(S_{1}\right)-\alpha D\right) X_{1}
\end{array}\right.
$$

Ce modèle a été étudié avec un taux de croissance $\mu_{1}$ non monotone et il a été établi que l'on peut avoir au plus quatre équilibres strictement positifs. Dans ce cas, le système peut présenter une bistabilité et pour toute condition initiale strictement positive, la solution converge vers un des équilibres strictement positifs [5]. Ce modèle a également été étudié pour un taux de croissance densité-dépendant $\mu_{0}\left(X_{0}, X_{1}\right)$ (par exemple lorsque la fonction de croissance est modélisée par la fonction de Contois), ce qui peut s'avérer intéressant si l'on travaille dans un environnement non homogène [6]. L'étude montre que le système présente soit une bistabilité soit une stabilité globale de l'équilibre strictement positif ou le lessivage. On fait l'hypothèse suivante :

H3 : La fonction $\mu_{0}(\cdot)$ est strictement croissante, $\mu_{0}(0)=0$ et $\mu_{0}^{\prime \prime}\left(X_{0}\right) \leqslant 0$ pour tout $\left.X_{0} \in\right] 0, \frac{X_{0 i n}}{\alpha}[$.

Dans le cas où la fonction $\mu_{0}(\cdot)$ est linéaire ou de type Monod, l'hypothèse $\mathbf{H 3}$ est vérifiée. On a le résultat suivant :

Proposition 3.1 Pour des valeurs initiales positives, les solutions du système (3) restent positives et bornées pour tout $t \geqslant 0$.

Preuve. Soit $X_{0}(0) \geqslant 0$, dès qu'il existe un premier temps $t_{0} \geqslant 0$ tel que $X_{0}\left(t_{0}\right)=0$, on a

$$
\dot{X}_{0}\left(t_{0}\right)=D X_{0 i n}>0 .
$$

D'où $X_{0}(t) \geqslant 0$ pour tout $t \geqslant t_{0}$. Comme $X_{0}(t) \geqslant 0$ pour tout $t \in\left[0, t_{0}\right]$, donc $X_{0}(t) \geqslant 0$ pour tout $t \geqslant 0$. Par le même raisonnement, on peut démontrer que toutes les solutions restent positives. Soit $Z=k_{0} X_{0}+S_{1}+k_{1} X_{1}$. En multipliant la première équation du système (3) par $k_{0}$ et la troisième équation par $k_{1}$, et faisant la somme des trois équations, on obtient

$$
\dot{Z}(t) \leqslant S_{\text {in }}-\alpha D Z(t)
$$

avec $S_{i n}=D\left(k_{0} X_{0 i n}+S_{1 i n}\right)$. On pose $V(t)=\alpha D Z(t)-S_{i n}$, alors

$$
\dot{V}(t) \leqslant-\alpha D V(t) \text {. }
$$

D'après le lemme de Gronwall, on a

$$
V(t) \leqslant V(0) e^{-\alpha D t}
$$

On en déduit que

$$
Z(t) \leqslant\left[\frac{S_{i n}}{\alpha D}+\left(Z(0)-\frac{S_{i n}}{\alpha D}\right) e^{-\alpha D t}\right] \leqslant \max \left(Z(0), \frac{S_{i n}}{\alpha D}\right), \text { pour tout } t \geqslant 0 .
$$

Par conséquent, les solutions sont bornées. 
Dans la suite, on se propose d'étudier l'existence des points d'équilibre du système (3) sous les hypothèses $\mathbf{H 1}$ et H3. L'équilibre de lessivage

$$
F_{0}=\left(\frac{X_{0 i n}}{\alpha}, S_{1 i n}, 0\right)
$$

existe toujours. Pour montrer l'existence des équilibres strictement positifs, on considère la fonction

$$
\xi\left(X_{0}\right)=\frac{D\left(X_{0 i n}-\alpha X_{0}\right)}{\mu_{0}\left(X_{0}\right)}
$$

et $\Delta$ la droite d'équation

$$
X_{1}=\delta\left(X_{0}\right)=\frac{1}{k_{1} \alpha}\left[\left(S_{1 i n}-\lambda_{1}\right)+k_{0}\left(X_{0 i n}-\alpha X_{0}\right)\right] .
$$

La recherche des équilibres strictement positifs revient à trouver les solutions de l'équation (voir Figs. 1, 2 et 3)

$$
\xi\left(X_{0}\right)=\delta\left(X_{0}\right)
$$

On étudie pour cela la fonction $H\left(X_{0}\right)=\xi\left(X_{0}\right)-\delta\left(X_{0}\right)$. La fonction $H^{\prime}\left(X_{0}\right)$ s'annule si et seulement si $\xi^{\prime}\left(X_{0}\right)=-\frac{k_{0}}{k_{1}}$.

Lemme 3.1 Sous l'hypothèse $\mathbf{H 3}$, la fonction $\xi(\cdot)$ s'annule en $\frac{X_{0 i n}}{\alpha}$, elle est décroissante et convexe.

Preuve. Pour tout $\left.X_{0} \in\right] 0, \frac{X_{0 i n}}{\alpha}[$, on a

$$
\xi^{\prime}\left(X_{0}\right)=-\frac{\alpha D}{\mu_{0}\left(X_{0}\right)}-\frac{\xi\left(X_{0}\right)}{\mu_{0}\left(X_{0}\right)} \mu_{0}^{\prime}\left(X_{0}\right)<0
$$

et

$$
\xi^{\prime \prime}\left(X_{0}\right)=-\frac{\xi\left(X_{0}\right)}{\mu_{0}\left(X_{0}\right)} \mu_{0}^{\prime \prime}\left(X_{0}\right)-2 \frac{\mu_{0}^{\prime}\left(X_{0}\right)}{\mu_{0}\left(X_{0}\right)} \xi^{\prime}\left(X_{0}\right)>0 .
$$

D'où $\xi(\cdot)$ est décroissante et est convexe sur $] 0, \frac{X_{0 i n}}{\alpha}[$.

Lemme 3.2 L'équation $\xi^{\prime}\left(X_{0}\right)=-\frac{k_{0}}{k_{1}}$ admet une unique solution $\left.\bar{X}_{0} \in\right] 0, \frac{X_{0 i n}}{\alpha}[$ si et seulement si

$$
\xi^{\prime}\left(\frac{X_{0 i n}}{\alpha}\right)>-\frac{k_{0}}{k_{1}}
$$

Preuve. D'après le Lemme 3.1, la fonction $\xi^{\prime}(\cdot)$ est strictement croissante sur $\left.] 0, \frac{X_{0 \text { in }}}{\alpha}\right]$. D'après (5), on déduit que

$$
\lim _{X_{0} \longrightarrow 0} \xi^{\prime}\left(X_{0}\right)=-\infty .
$$

Par suite, il existe une unique solution $\left.\bar{X}_{0} \in\right] 0, \frac{X_{0 i n}}{\alpha}\left[\right.$ de l'équation $\xi^{\prime}\left(X_{0}\right)=-\frac{k_{0}}{k_{1}}$ si et seulement si

$$
\xi^{\prime}\left(\frac{X_{0 i n}}{\alpha}\right)>-\frac{k_{0}}{k_{1}} \text {. }
$$


On peut facilement vérifier l'équivalence suivante, qui donne deux cas possibles sur l'existence des équilibres strictement positifs du sous modèle (3)

$$
\xi^{\prime}\left(\frac{X_{0 i n}}{\alpha}\right)>-\frac{k_{0}}{k_{1}} \Longleftrightarrow k_{0} \mu_{0}\left(\frac{X_{0 i n}}{\alpha}\right)>k_{1} \alpha D .
$$

3.1. Le cas $\xi^{\prime}\left(\frac{X_{0 i n}}{\alpha}\right) \leqslant-\frac{k_{0}}{k_{1}}$

On commence par montrer l'existence d'au plus un point équilibre strictement positif suivant la concentration du substrat à l'entrée du chémostat $S_{1 \text { in }}$ dans le cas où $\xi^{\prime}\left(\frac{X_{0 i n}}{\alpha}\right) \leqslant-\frac{k_{0}}{k_{1}}$ (voir Fig. 1). Notons que, dans ce cas, l'intersection de la droite $\Delta$ avec le graphe de la fonction $\xi$ est soit vide, soit un unique point qu' on notera $F_{1}^{*}=\left(X_{0}^{*}, X_{1}^{*}\right)$. En effet pour ne pas multiplier et alourdir les notations, on notera par la même lettre un équilibre $F^{*}=\left(X_{0}^{*}, \lambda_{1}, X_{1}^{*}\right)$ du système (3) et sa projection $F^{*}=\left(X_{0}^{*}, X_{1}^{*}\right)$ dans le plan $\left(X_{0}, X_{1}\right)$.

\section{Proposition 3.2}

1) Si $S_{1 i n}>\lambda_{1}$, alors il existe un unique équilibre strictement positif

$$
F_{1}^{*}=\left(X_{0}^{*}, \lambda_{1}, X_{1}^{*}\right)
$$

avec $X_{0}^{*}$ est solution de l'équation $\xi\left(X_{0}\right)=\delta\left(X_{0}\right)$ et $X_{1}^{*}=\delta\left(X_{0}^{*}\right)$ (voir Fig. $1(a)$ ).

2) Si $S_{1 i n} \leqslant \lambda_{1}$, alors il n'existe pas d'équilibre strictement positif(voir Fig. 1 (b) et $(c))$.

Preuve. D'après le Lemme 3.1, on a

$$
\left.\xi^{\prime}\left(X_{0}\right)<\xi^{\prime}\left(\frac{X_{0 i n}}{\alpha}\right) \leqslant-\frac{k_{0}}{k_{1}}, \quad \text { pour tout } \quad X_{0} \in\right] 0, \frac{X_{0 i n}}{\alpha}[
$$

c'est-à-dire $H^{\prime}\left(X_{0}\right)=\xi^{\prime}\left(X_{0}\right)+\frac{k_{0}}{k_{1}}<0$. De plus,

$$
\lim _{X_{0} \rightarrow 0} H\left(X_{0}\right)=+\infty
$$

Si $S_{1 i n}>\lambda_{1}$, alors $H\left(\frac{X_{0 i n}}{\alpha}\right)=-\delta\left(\frac{X_{0 i n}}{\alpha}\right)<0$. Par suite, il existe une unique solution $\left.X_{0}^{*} \in\right] 0, \frac{X_{0 i n}}{\alpha}\left[\right.$ de l'équation $\xi\left(X_{0}\right)=\delta\left(X_{0}\right)$ avec $X_{1}^{*}=\delta\left(X_{0}^{*}\right)$ (voir Fig. 1 (a)).

Si $S_{1 \text { in }} \leqslant \lambda_{1}$, alors $H\left(\frac{X_{0 i n}}{\alpha}\right) \geqslant 0$ et comme $H$ est strictement décroissante alors l'équation $\xi\left(X_{0}\right)=\delta\left(X_{0}\right)$ n'admet pas de solution sur $] 0, \frac{X_{0 i n}}{\alpha}[$ et l'équilibre strictement positif n'existe pas (voir Fig. 1 (b) et (c)).

On remarque que lorsque $S_{1 i n}=\lambda_{1}, F_{1}^{*}$ bifurque avec $F_{0}$. On choisit la couleur rouge pour le point d'équilibre LAS, la couleur bleue pour le point d'équilibre instable et la couleur verte pour le point d'équilibre nœud col (voir Figs. 1-6). On montrera le comportement asymptotique des points d'équilibre du sous-modèle (3) dans les Propositions 3.4 et 3.5 . 

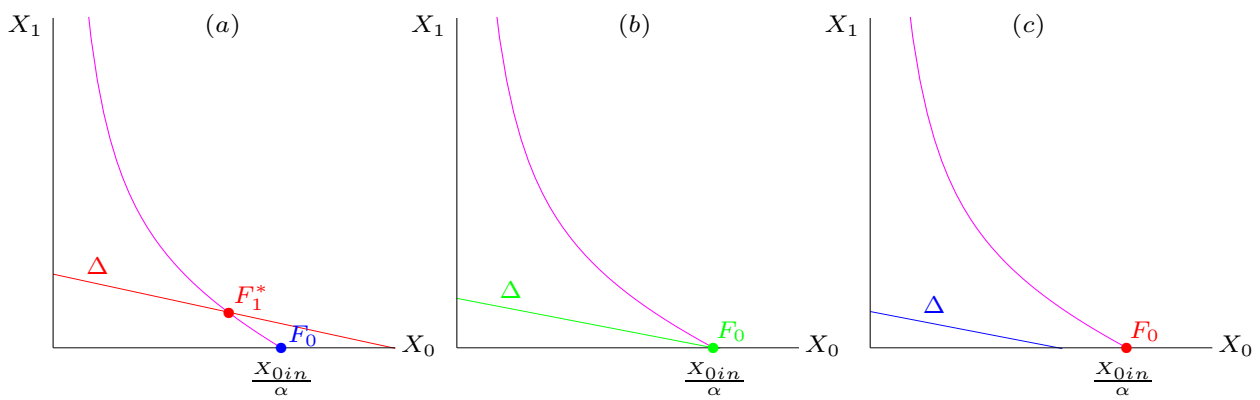

Figure 1. Existence des points d'équilibre selon le paramètre $S_{1 i n}$. (a) $S_{1 i n}>\lambda_{1}$. (b) $S_{1 \text { in }}=\lambda_{1}$. (c) $S_{1 \text { in }}<\lambda_{1}$.

\subsection{Le cas $\xi^{\prime}\left(\frac{X_{0 i n}}{\alpha}\right)>-\frac{k_{0}}{k_{1}}$}

Dans ce cas, on démontre que l'intersection de la droite $\Delta$ avec le graphe de la fonction $\xi$, si elle n'est pas vide, comporte un ou deux points qu'on notera $F_{1}^{*}=\left(X_{0}^{*}, X_{1}^{*}\right)$ et $F_{1}^{* *}=\left(X_{0}^{* *}, X_{1}^{* *}\right)$ (voir Fig. 2). Ces points correspondent respectivement aux équilibres $F_{1}^{*}=\left(X_{0}^{*}, \lambda_{1}, X_{1}^{*}\right)$ et $F_{1}^{* *}=\left(X_{0}^{* *}, \lambda_{1}, X_{1}^{* *}\right)$ de (3) que l'on notera par les mêmes lettres $F_{1}^{*}$ et $F_{1}^{* *}$. L'équation $\xi^{\prime}\left(X_{0}\right)=-\frac{k_{0}}{k_{1}}$ admet une unique solution notée $\bar{X}_{0}$. On pose $\bar{X}_{1}=\xi\left(\bar{X}_{0}\right)$. Noter que

$$
\bar{F}_{1}=\left(\bar{X}_{0}, \lambda_{1}, \bar{X}_{1}\right)
$$

est un équilibre strictement positif du système (3) (voir Fig. 3 (d)). Soit $\bar{S}_{1 \text { in }}$ la valeur de $S_{1 \text { in }}$ pour laquelle

$$
\bar{X}_{1}=\delta\left(\bar{X}_{0}\right)=-\frac{k_{0}}{k_{1}} \bar{X}_{0}+B \quad \text { avec } \quad B=\delta(0)=\frac{1}{k_{1} \alpha}\left[\left(\bar{S}_{1 i n}-\lambda_{1}\right)+k_{0} X_{0 i n}\right] .
$$

D'où

$$
\bar{S}_{1 i n}=\alpha\left(k_{1} \bar{X}_{1}+k_{0} \bar{X}_{0}\right)+\lambda_{1}-k_{0} X_{0 i n} .
$$

\section{Remarque.}
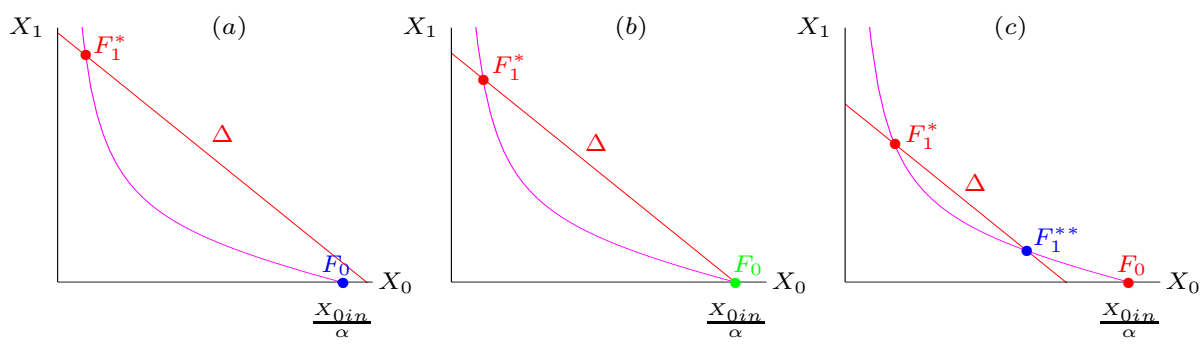

Figure 2. Existence des points d'équilibre selon le paramètre $S_{1 \text { in }}$. (a) $\lambda_{1}<S_{1 \text { in }}$. (b) $S_{1 \text { in }}=\lambda_{1}$. (c) $\max \left(0, \bar{S}_{1 \text { in }}\right)<S_{1 \text { in }}<\lambda_{1}$.

Si $\bar{S}_{1 \text { in }}<0$, alors le cas limite de la droite obtenu pour $S_{1 i n}=0$ se trouve dans (c). Si $\bar{S}_{1 i n}=0$, alors le cas limite de la droite obtenu pour $S_{1 i n}=0$ se trouve dans (d). Si $\bar{S}_{1 \text { in }}>0$, alors le cas limite de la droite obtenu pour $S_{1 i n}=0$ se trouve dans (e). Le dernier cas (e) pouvant être vide lorsque $\bar{S}_{1 \text { in }} \leqslant 0$. 

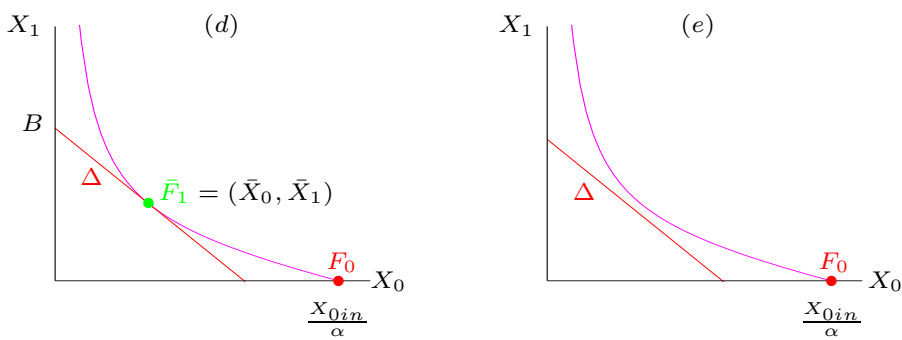

Figure 3. (d) $S_{1 i n}=\bar{S}_{1 i n}$. (e) $0<S_{1 \text { in }}<\max \left(0, \bar{S}_{1 i n}\right)$.

\section{Proposition 3.3}

1) Si $\lambda_{1} \leqslant S_{1 i n}$, alors il existe un unique équilibre strictement positif $F_{1}^{*}$ (voir Fig. $2(a)$ et $(b))$.

2) Si $\max \left(0, \bar{S}_{1 \text { in }}\right)<S_{1 \text { in }}<\lambda_{1}$, alors il existe deux équilibres strictement positifs $F_{1}^{*}$ et $F_{1}^{* *}$ (voir Fig. $2(c)$ ).

3) Si $S_{1 i n}=\bar{S}_{1 i n}$, alors il existe un unique équilibre strictement positif $\bar{F}_{1}$ (voir Fig. $3(d)$ ).

4) Si $S_{1 \text { in }}<\max \left(0, \bar{S}_{1 \text { in }}\right)$, alors il n'existe aucun équilibre strictement positif (voir Fig. $3(e)$ ).

Preuve. D'après le Lemme 3.1, la fonction $H(\cdot)$ est convexe et

$$
\lim _{X_{0} \rightarrow 0} H\left(X_{0}\right)=+\infty .
$$

Si $S_{1 i n} \geqslant \lambda_{1}$, alors $H\left(\frac{X_{0 i n}}{\alpha}\right) \leqslant 0$. Par suite, il existe une unique solution $\left.X_{0}^{*} \in\right] 0, \frac{X_{0 i n}}{\alpha}[$ de l'équation $H\left(X_{0}\right)=0$ (voir Fig. 2 (a) et (b)).

Pour $S_{1 i n}=\bar{S}_{1 i n}$, on considère la fonction

$$
\bar{H}\left(X_{0}\right)=\xi\left(X_{0}\right)-\frac{1}{k_{1} \alpha}\left[\left(\bar{S}_{1 i n}-\lambda_{1}\right)+k_{0}\left(X_{0 i n}-\alpha X_{0}\right)[.\right.
$$

Comme $\bar{H}^{\prime}\left(X_{0}\right)=H^{\prime}\left(X_{0}\right)$, alors d'après le Lemme 3.2, il existe une unique solution $\left.\bar{X}_{0} \in\right] 0, \frac{X_{0 i n}}{\alpha}\left[\right.$ de l'équation $\bar{H}^{\prime}\left(X_{0}\right)=0$. De plus, $\bar{H}(\cdot)$ est convexe et

$$
\bar{H}\left(\bar{X}_{0}\right)=0=\min _{X \in] 0, \frac{X_{0 i n}}{\alpha}[} \bar{H}(X) .
$$

D'où l'équation $\bar{H}\left(X_{0}\right)=0$ admet une unique solution $\left.\bar{X}_{0} \in\right] 0, \frac{X_{0 i n}}{\alpha}[$ (voir Fig. 3 (d)). Si $S_{1 \text { in }}<\max \left(0, \bar{S}_{1 i n}\right)$, alors

$$
\left.H\left(X_{0}\right)>\bar{H}\left(X_{0}\right) \geqslant \min _{X \in] 0, \frac{X_{0 i n}}{\alpha}[} \bar{H}(X)=0, \quad \text { pour tout } \quad X_{0} \in\right] 0, \frac{X_{0 i n}}{\alpha}[.
$$

Par conséquent, l'équation $H\left(X_{0}\right)=0$ n'admet pas de solution (voir Fig. 3 (e)). Si $\max \left(0, \bar{S}_{1 i n}\right)<S_{1 i n}$, alors

$$
\min _{X \in] 0, \frac{X_{0 i n}}{\alpha}[} H(X)<\min _{X \in] 0, \frac{X_{0 i n}}{\alpha}[} \bar{H}(X)=0 .
$$

De plus, $H(\cdot)$ est convexe et pour $S_{1 \text { in }}<\lambda_{1}$, on a $H\left(\frac{X_{0 i n}}{\alpha}\right)>0$. Par suite, il existe deux solutions $X_{0}^{*}$ et $X_{0}^{* *}$ dans $] 0, \frac{X_{0 i n}}{\alpha}\left[\right.$ de l'équation $H\left(X_{0}\right)=0$ (voir Fig. 2 (c)). 


\subsection{Stabilité locale}

On se propose, ici, d'étudier la stabilité locale des points d'équilibre du système (3). La matrice jacobienne en $\left(X_{0}, S_{1}, X_{1}\right)$ est :

$$
\mathbf{J}=\left[\begin{array}{ccc}
-\left[\alpha D+\mu_{0}^{\prime}\left(X_{0}\right) X_{1}\right] & 0 & -\mu_{0}\left(X_{0}\right) \\
k_{0} \mu_{0}^{\prime}\left(X_{0}\right) X_{1} & -\left[D+k_{1} \mu_{1}^{\prime}\left(S_{1}\right) X_{1}\right] & k_{0} \mu_{0}\left(X_{0}\right)-k_{1} \mu_{1}\left(S_{1}\right) \\
0 & \mu_{1}^{\prime}\left(S_{1}\right) X_{1} & \mu_{1}\left(S_{1}\right)-\alpha D
\end{array}\right]
$$

Proposition 3.4 $F_{0}$ est LAS si et seulement si $\mu_{1}\left(S_{1 i n}\right)<\alpha D$.

Preuve. La matrice jacobienne en $F_{0}=\left(\frac{X_{0 i n}}{\alpha}, S_{1 i n}, 0\right)$ est égale à

$$
\mathbf{J}_{\mathbf{0}}=\left[\begin{array}{ccc}
-\alpha D & 0 & -\mu_{0}\left(\frac{X_{0 i n}}{\alpha}\right) \\
0 & -D & k_{0} \mu_{0}\left(\frac{X_{0 i n}}{\alpha}\right)-k_{1} \mu_{1}\left(S_{1 i n}\right) \\
0 & 0 & \mu_{1}\left(S_{1 i n}\right)-\alpha D
\end{array}\right]
$$

Les valeurs propres sont $-\alpha D,-D$ et $\mu_{1}\left(S_{1 \text { in }}\right)-\alpha D$. Ces valeurs propres sont strictement négatives si et seulement si $\mu_{1}\left(S_{1 i n}\right)-\alpha D<0$.

\section{Proposition 3.5}

1) Si $F_{1}^{*}$ existe, alors il est LAS.

2) Si $F_{1}^{* *}$ existe, alors il est instable.

Preuve. La matrice jacobienne en $F_{1}^{*}=\left(X_{0}^{*}, \lambda_{1}, X_{1}^{*}\right)$ est égale à

$$
\mathbf{J}_{\mathbf{1}}=\left[\begin{array}{ccc}
-m_{11} & 0 & -m_{13} \\
m_{21} & -m_{22} & \theta \\
0 & m_{32} & 0
\end{array}\right]
$$

où

$$
\begin{gathered}
m_{11}=\alpha D+\mu_{0}^{\prime}\left(X_{0}^{*}\right) X_{1}^{*}, \quad m_{13}=\mu_{0}\left(X_{0}^{*}\right), \quad m_{21}=k_{0} \mu_{0}^{\prime}\left(X_{0}^{*}\right) X_{1}^{*}, \\
m_{22}=D+k_{1} \mu_{1}^{\prime}\left(\lambda_{1}\right) X_{1}^{*}, \quad \theta=k_{0} \mu_{0}\left(X_{0}^{*}\right)-k_{1} \alpha D, \quad m_{32}=\mu_{1}^{\prime}\left(\lambda_{1}\right) X_{1}^{*}
\end{gathered}
$$

avec $m_{11}, m_{13}, m_{21}, m_{22}$ et $m_{32}$ sont strictement positifs. Le polynôme caractéristique de $J_{1}$ est donné par

$$
P_{J_{1}}(\lambda)=a_{0} \lambda^{3}+a_{1} \lambda^{2}+a_{2} \lambda+a_{3}
$$

avec

$a_{0}=-1, a_{1}=-\left(m_{11}+m_{22}\right), a_{2}=-m_{11} m_{22}+\theta m_{32}, a_{3}=-m_{32}\left(m_{21} m_{13}-\theta m_{11}\right)$.

D'après le critère de Routh-Hurwitz, $F_{1}^{*}$ est LAS si et seulement si

$$
\left\{\begin{array}{l}
a_{i}<0, \\
a_{1} a_{2}-a_{0} a_{3}>0 .
\end{array} \quad i=0, \ldots, 3\right.
$$

On peut facilement vérifier que

$$
\begin{aligned}
a_{2} & =\left[k_{0} \mu_{0}\left(X_{0}^{*}\right)-k_{1} \alpha D\right] m_{32}-\left[\alpha D+\mu_{0}^{\prime}\left(X_{0}^{*}\right) X_{1}^{*}\right]\left[D+k_{1} m_{32}\right] \\
& =\left[k_{0} \mu_{0}\left(X_{0}^{*}\right)-k_{1} m_{11}\right] m_{32}-P
\end{aligned}
$$


avec $P=\alpha D m_{22}+D \mu_{0}^{\prime}\left(X_{0}^{*}\right) X_{1}^{*}$, qui est strictement positif. En remplaçant $\xi^{\prime}\left(X_{0}\right)$ par son expression (5), on obtient

$$
\xi^{\prime}\left(X_{0}^{*}\right)+\frac{k_{0}}{k_{1}}=\frac{k_{0} \mu_{0}\left(X_{0}^{*}\right)-k_{1} m_{11}}{k_{1} \mu_{0}\left(X_{0}^{*}\right)}
$$

Par suite,

$$
a_{2}=\left[\xi^{\prime}\left(X_{0}^{*}\right)+\frac{k_{0}}{k_{1}}\right] k_{1} \mu_{0}\left(X_{0}^{*}\right) m_{32}-P .
$$

Si $\xi^{\prime}\left(X_{0}^{*}\right)<-\frac{k_{0}}{k_{1}}$, alors $a_{2}<0$. De plus,

$$
a_{3}=m_{32} \alpha D\left[k_{0} \mu_{0}\left(X_{0}^{*}\right)-k_{1} m_{11}\right]=m_{32} \alpha D\left[\xi^{\prime}\left(X_{0}^{*}\right)+\frac{k_{0}}{k_{1}}\right] k_{1} \mu_{0}\left(X_{0}^{*}\right)
$$

qui est strictement négatif si et seulement si $\xi^{\prime}\left(X_{0}^{*}\right)<-\frac{k_{0}}{k_{1}}$. Finalement,

$$
\begin{aligned}
a_{1} a_{2}-a_{0} a_{3} & =-m_{11} a_{2}+a_{3}-m_{22} a_{2} \\
& =-\left[\alpha D+\mu_{0}^{\prime}\left(X_{0}^{*}\right) X_{1}^{*}\right] a_{2}+a_{3}-m_{22} a_{2} \\
& =\alpha D P-\mu_{0}^{\prime}\left(X_{0}^{*}\right) X_{1}^{*} a_{2}-m_{22} a_{2}
\end{aligned}
$$

qui est strictement positif si $\xi^{\prime}\left(X_{0}^{*}\right)<-\frac{k_{0}}{k_{1}}$. Comme $F_{1}^{*}$ vérifie $\xi^{\prime}\left(X_{0}^{*}\right)<-\frac{k_{0}}{k_{1}}$, alors il est LAS et $F_{1}^{* *}$ vérifie $\xi^{\prime}\left(X_{0}^{* *}\right)>-\frac{k_{0}}{k_{1}}$, alors il est instable, ce qui achève la preuve de la proposition.

\section{Etude du modèle complet à trois étapes}

Dans la suite, on se propose d'étudier le modèle à trois étapes (1) avec $r_{0}=\mu_{0}\left(X_{0}\right) X_{1}$ sous les hypothèses H1-H3. Le modèle s'écrit

$$
\left\{\begin{aligned}
\dot{X}_{0} & =D X_{0 i n}-\alpha D X_{0}-\mu_{0}\left(X_{0}\right) X_{1} \\
\dot{S}_{1} & =D\left(S_{1 i n}-S_{1}\right)+k_{0} \mu_{0}\left(X_{0}\right) X_{1}-k_{1} \mu_{1}\left(S_{1}\right) X_{1} \\
\dot{X}_{1} & =\left(\mu_{1}\left(S_{1}\right)-\alpha D\right) X_{1} \\
\dot{S}_{2} & =D\left(S_{2 i n}-S_{2}\right)+k_{2} \mu_{1}\left(S_{1}\right) X_{1}-k_{3} \mu_{2}\left(S_{2}\right) X_{2} \\
\dot{X}_{2} & =\left(\mu_{2}\left(S_{2}\right)-\alpha D\right) X_{2} .
\end{aligned}\right.
$$

\subsection{Existence des points d'équilibre}

Dans le cas $\xi^{\prime}\left(\frac{X_{0 i n}}{\alpha}\right) \leqslant-\frac{k_{0}}{k_{1}}$ ou $\xi^{\prime}\left(\frac{X_{0 i n}}{\alpha}\right)>-\frac{k_{0}}{k_{1}}$, on démontre le résultat suivant :

Proposition 4.1 Supposons que $\lambda_{1} \leqslant S_{1 i n}$. Les points d'équilibre du système (8) sont donnés par

1) $E_{0}=\left(\frac{X_{0 i n}}{\alpha}, S_{1 i n}, 0, S_{2 i n}, 0\right)$ qui existe toujours.

2) $E_{1}^{i}=\left(\frac{X_{0 i n}}{\alpha}, S_{1 i n}, 0, \lambda_{2}^{i}, X_{2}^{i}\right), i=1,2$, avec $X_{2}^{i}=\frac{1}{k_{3} \alpha}\left(S_{2 i n}-\lambda_{2}^{i}\right)$, qui existe si et seulement si $S_{2 i n}>\lambda_{2}^{i}$. 
3) $E_{2}^{0 *}=\left(X_{0}^{*}, \lambda_{1}, X_{1}^{*}, S_{2 i n}^{*}, 0\right)$ avec $X_{0}^{*}$ l'unique solution de $\xi\left(X_{0}\right)=\delta\left(X_{0}\right)$ et

$$
X_{1}^{*}=\delta\left(X_{0}^{*}\right), \quad S_{2 i n}^{*}=S_{2 i n}+\alpha k_{2} X_{1}^{*},
$$

qui existe toujours.

4) $E_{2}^{i *}=\left(X_{0}^{*}, \lambda_{1}, X_{1}^{*}, \lambda_{2}^{i}, X_{2}^{i *}\right), i=1,2$, avec

$$
X_{2}^{i *}=\frac{1}{k_{3} \alpha}\left(S_{2 i n}-\lambda_{2}^{i}\right)+\frac{k_{2}}{k_{3}} X_{1}^{*},
$$

qui existe si et seulement si $S_{2 i n}^{*}>\lambda_{2}^{i}$.

Preuve. À chaque équilibre $F=\left(X_{0}, S_{1}, X_{1}\right)$ de (3) correspond un équilibre

$$
E=\left(X_{0}, S_{1}, X_{1}, S_{2}, X_{2}\right)
$$

où $\left(X_{0}, S_{1}, X_{1}\right)$ sont les composantes de l'équilibre $F$ et $\left(S_{2}, X_{2}\right)$ les solutions du système

$$
\begin{aligned}
0 & =D\left(S_{2 i n}-S_{2}\right)+k_{2} \mu_{1}\left(S_{1}\right) X_{1}-k_{3} \mu_{2}\left(S_{2}\right) X_{2}, \\
0 & =\left(\mu_{2}\left(S_{2}\right)-\alpha D\right) X_{2} .
\end{aligned}
$$

De la deuxième équation on déduit que soit $X_{2}=0$, soit $S_{2}=\lambda_{2}^{i}$.

Si $X_{2}=0$, de la première équation on déduit alors que

$$
S_{2}=S_{2 i n}+\frac{k_{2}}{D} \mu_{1}\left(S_{1}\right) X_{1}
$$

Cette construction permet d'obtenir l'équilibre $E_{0}$ à partir de l'équilibre $F_{0}$ de (3), donné par (4), et d'obtenir l'équilibre $E_{2}^{0 *}$ à partir de l'équilibre $F_{1}^{*}$ de (3), donné par (6).

$\mathrm{Si} S_{2}=\lambda_{2}^{i}$, de la première équation on déduit alors que

$$
X_{2}=\frac{1}{k_{3} \alpha}\left(S_{2 i n}-\lambda_{2}^{i}\right)+\frac{k_{2}}{k_{3} \alpha D} \mu_{1}\left(S_{1}\right) X_{1} .
$$

Cette construction permet d'obtenir les équilibres $E_{1}^{i}$ à partir de l'équilibre $F_{0}$ de (3), donné par (4), et d'obtenir les équilibres $E_{2}^{i *}$ à partir de l'équilibre $F_{1}^{*}$ de (3), donné par (6).

De la même manière on démontre le résultat suivant dans le cas générique où le sousmodèle (3) peut avoir deux équilibres strictement positifs, c'est à dire pour

$$
\xi^{\prime}\left(\frac{X_{0 i n}}{\alpha}\right)>-\frac{k_{0}}{k_{1}}
$$

Proposition 4.2 Supposons que $\max \left(0, \bar{S}_{1 \text { in }}\right)<S_{1 \text { in }}<\lambda_{1}$. Les points d'équilibre du système (8) sont donnés par

1) $E_{0}=\left(\frac{X_{0 i n}}{\alpha}, S_{1 i n}, 0, S_{2 i n}, 0\right)$ qui existe toujours.

2) $E_{1}^{i}=\left(\frac{X_{0 i n}}{\alpha}, S_{1 i n}, 0, \lambda_{2}^{i}, X_{2}^{i}\right), i=1,2$, avec $X_{2}^{i}=\frac{1}{k_{3} \alpha}\left(S_{2 i n}-\lambda_{2}^{i}\right)$, qui existe si et seulement si $S_{2 \text { in }}>\lambda_{2}^{i}$. 
3) $E_{2}^{0 *}=\left(X_{0}^{*}, \lambda_{1}, X_{1}^{*}, S_{2 i n}^{*}, 0\right)$ et $E_{2}^{0 * *}=\left(X_{0}^{* *}, \lambda_{1}, X_{1}^{* *}, S_{2 i n}^{* *}, 0\right)$ avec $X_{0}^{*}$ et $X_{0}^{* *}$ les deux solutions de l'équation $\xi\left(X_{0}\right)=\delta\left(X_{0}\right)$ et

$$
\begin{gathered}
X_{1}^{*}=\delta\left(X_{0}^{*}\right), \quad S_{2 i n}^{*}=S_{2 i n}+\alpha k_{2} X_{1}^{*}, \\
X_{1}^{* *}=\delta\left(X_{0}^{* *}\right), \quad S_{2 i n}^{* *}=S_{2 i n}+\alpha k_{2} X_{1}^{* *},
\end{gathered}
$$

qui existent toujours.

4) $E_{2}^{i *}=\left(X_{0}^{*}, \lambda_{1}, X_{1}^{*}, \lambda_{2}^{i}, X_{2}^{* i}\right)$ et $E_{2}^{i * *}=\left(X_{0}^{* *}, \lambda_{1}, X_{1}^{* *}, \lambda_{2}^{i}, X_{2}^{i * *}\right), i=1,2$, avec

$$
X_{2}^{i *}=\frac{1}{k_{3} \alpha}\left(S_{2 i n}-\lambda_{2}^{i}\right)+\frac{k_{2}}{k_{3}} X_{1}^{*} \quad \text { et } \quad X_{2}^{i * *}=\frac{1}{k_{3} \alpha}\left(S_{2 i n}-\lambda_{2}^{i}\right)+\frac{k_{2}}{k_{3}} X_{1}^{* *},
$$

qui existent si et seulement si $S_{2 i n}^{*}>\lambda_{2}^{i}$ et $S_{2 i n}^{* *}>\lambda_{2}^{i}$.

Par conséquent, le modèle à trois étapes (8) peut avoir au plus neuf points d'équilibre et au plus quatre points d'équilibre strictement positifs où les deux espèces coexistent.

\subsection{Stabilité locale}

On se propose d'étudier la stabilité locale des points d'équilibre du système (8). Comme le système est triangulaire, la matrice jacobienne en un équilibre $E=\left(X_{0}, S_{1}, X_{1}, S_{2}, X_{2}\right)$ est de la forme :

$$
\mathbf{J}_{\mathbf{E}}=\left[\begin{array}{cc}
J_{F} & 0 \\
B & C
\end{array}\right]
$$

avec $J_{F}$ la matrice jacobienne de (3) en l'équilibre $F=\left(X_{0}, S_{1}, X_{1}\right), B$ un matrice $2 \times 3$ et $C$ une matrice carrée d'ordre 2. Comme la matrice $J_{E}$ est triangulaire inférieure par blocs, elle est stable si et seulement si les matrices $J_{F}$ et $C$ sont stables. La stabilité de la matrice $J_{F}$ a été étudiée dans la section 3.3. Le problème revient donc à étudier la stabilité de la matrice d'ordre 2, c'est à dire à voir si sa trace est négative et son déterminant est positif.

Proposition 4.3 $E_{0}$ est LAS si et seulement si $\mu_{1}\left(S_{1 i n}\right)<\alpha D$ et $\mu_{2}\left(S_{2 i n}\right)<\alpha D$.

Preuve. En $E_{0}$ on obtient $J_{F}=J_{0}$, où $J_{0}$ est la matrice considérée dans la preuve de la Proposition 3.4 et

$$
C=\left[\begin{array}{cc}
-D & -k_{3} \mu_{2}\left(S_{2 i n}\right) \\
0 & \mu_{2}\left(S_{2 i n}\right)-\alpha D
\end{array}\right] \text {. }
$$

Noter que $J_{0}$ est stable si et seulement si $\mu_{1}\left(S_{1 \text { in }}\right)<\alpha D$ et $C$ est stable si et seulement si $\mu_{2}\left(S_{2 i n}\right)<\alpha D$.

Proposition 4.4 Si $E_{1}^{1}$ existe, alors il est LAS si et seulement si $\mu_{1}\left(S_{1 i n}\right)<\alpha D$. Si $E_{1}^{2}$ existe, alors il est instable.

Preuve. En $E_{1}^{i}$ on obtient $J_{F}=J_{0}$, où $J_{0}$ est la matrice considérée dans la preuve de la Proposition 3.4 et

$$
\mathbf{C}=\left[\begin{array}{cc}
-\left[D+k_{3} \mu_{2}^{\prime}\left(\lambda_{2}^{i}\right) X_{2}^{i}\right] & -k_{3} \alpha D \\
\mu_{2}^{\prime}\left(\lambda_{2}^{i}\right) X_{2}^{i} & 0
\end{array}\right]
$$


On a

$$
\operatorname{det} C=k_{3} \alpha D \mu_{2}^{\prime}\left(\lambda_{2}^{i}\right) X_{2}^{i} \quad \text { et } \quad \operatorname{tr} C=-\left[D+k_{3} \mu_{2}^{\prime}\left(\lambda_{2}^{i}\right) X_{2}^{i}\right] .
$$

Comme $\operatorname{det} C<0$ pour $i=2$, alors dès qu'il existe l'équilibre $E_{1}^{2}$, il est instable. Noter que $J_{0}$ est stable si et seulement si $\mu_{1}\left(S_{1 i n}\right)<\alpha D$. Pour $i=1$, on a $\operatorname{det} C>0$ et $\operatorname{tr} C<0$. Par suite, l'équilibre $E_{1}^{1}$ est LAS si et seulement si $\mu_{1}\left(S_{1 i n}\right)<\alpha D$.

Proposition 4.5 Si $E_{2}^{0 *}$ existe, alors il est LAS si et seulement si $\mu_{2}\left(S_{2 i n}^{*}\right)<\alpha D$. Si $E_{2}^{0 * *}$ existe, alors il est instable.

Preuve. En $E_{2}^{0 *}$ on obtient $J_{F}=J_{1}$, où $J_{1}$ est la matrice considérée dans la preuve de la Proposition 3.5 et

$$
\mathbf{C}=\left[\begin{array}{cc}
-D & -k_{3} \mu_{2}\left(S_{2 i n}^{*}\right) \\
0 & \mu_{2}\left(S_{2 i n}^{*}\right)-\alpha D
\end{array}\right]
$$

Noter que $J_{1}$ est stable et $C$ est stable si et seulement si $\mu_{2}\left(S_{2 i n}^{*}\right)<\alpha D$. En $E_{2}^{0 * *}$ on obtient $J_{F}=J_{1}$, où $J_{1}$ est une matrice instable d'après la preuve de la Proposition 3.5.

Proposition 4.6 Si $E_{2}^{1 *}$ existe, alors il est LAS. Si $E_{2}^{2 *}$ et $E_{2}^{i * *}, i=1,2$, existent, alors ils sont instables.

Preuve. En $E_{2}^{i *}$ on obtient $J_{F}=J_{1}$, où $J_{1}$ est la matrice considérée dans la preuve de la Proposition 3.5 et

$$
\mathbf{C}=\left[\begin{array}{cc}
-\left[D+k_{3} \mu_{2}^{\prime}\left(\lambda_{2}^{i}\right) X_{2}^{i *}\right] & -k_{3} \alpha D \\
\mu_{2}^{\prime}\left(\lambda_{2}^{i}\right) X_{2}^{i *} & 0
\end{array}\right]
$$

Comme $\operatorname{det} C<0$ pour $i=2$, alors dès qu'il existe l'équilibre strictement positif $E_{2}^{2 *}$, il est instable. Noter que $J_{1}$ est stable, $\operatorname{det} C>0$ et $\operatorname{tr} C<0$ pour $i=1$. Par suite, dès qu'il existe l'équilibre strictement positif $E_{2}^{1 *}$, il est LAS. En $E_{2}^{i * *}$ on obtient $J_{F}=J_{1}$, où $J_{1}$ est une matrice instable d'après la preuve de la Proposition 3.5.

Le tableau suivant résume les résultats des Propositions 4.3-4.6 dans le cas $S_{1 \text { in }}>\lambda_{1}$, où la lettre $\mathrm{S}$ signifie que l'équilibre est stable et la lettre I signifie que l'équilibre est instable. L'absence de lettre signifie que l'équilibre n'existe pas.

\begin{tabular}{lcccccc}
\hline Condition & \multicolumn{5}{c}{ Équilibres et nature } \\
\cline { 2 - 7 } & $E_{0}$ & $E_{1}^{1}$ & $E_{1}^{2}$ & $E_{2}^{0 *}$ & $E_{2}^{1 *}$ & $E_{2}^{2 *}$ \\
\hline 1) $\lambda_{2}^{1}<\lambda_{2}^{2}<S_{2 i n}<S_{2 i n}^{*}$ & $\mathrm{I}$ & $\mathrm{I}$ & $\mathrm{I}$ & $\mathrm{S}$ & $\mathrm{S}$ & $\mathrm{I}$ \\
2) $\lambda_{2}^{1}<S_{2 i n}<S_{2 i n}^{*}<\lambda_{2}^{2}$ & $\mathrm{I}$ & $\mathrm{I}$ & & $\mathrm{I}$ & $\mathrm{S}$ & \\
3) $\lambda_{2}^{1}<S_{2 i n}<\lambda_{2}^{2}<S_{2 i n}^{*}$ & $\mathrm{I}$ & $\mathrm{I}$ & & $\mathrm{S}$ & $\mathrm{S}$ & $\mathrm{I}$ \\
4) $S_{2 i n}<\lambda_{2}^{1}<\lambda_{2}^{2}<S_{2 i n}^{*}$ & $\mathrm{I}$ & & & $\mathrm{S}$ & $\mathrm{S}$ & $\mathrm{I}$ \\
5) $S_{2 i n}<\lambda_{2}^{1}<S_{2 i n}^{*}<\lambda_{2}^{2}$ & $\mathrm{I}$ & & & $\mathrm{I}$ & $\mathrm{S}$ & \\
6) $S_{2 i n}<S_{2 i n}^{*}<\lambda_{2}^{1}<\lambda_{2}^{2}$ & $\mathrm{I}$ & & $\mathrm{S}$ & & \\
\hline
\end{tabular}


Comme $X_{0}^{*}<X_{0}^{* *}$, alors $X_{1}^{*}>X_{1}^{* *}$ et par suite, $S_{2 i n}<S_{2 i n}^{* *}<S_{2 i n}^{*}$. On obtient dans le cas $\max \left(0, \bar{S}_{1 \text { in }}\right)<S_{1 \text { in }}<\lambda_{1}$ le tableau suivant :

\begin{tabular}{|c|c|c|c|c|c|c|c|c|c|}
\hline \multirow[t]{2}{*}{ Condition } & \multicolumn{6}{|c|}{ Équilibres et nature } & \multirow[b]{2}{*}{$E_{2}^{1 * *}$} & \multirow[b]{2}{*}{$E_{2}^{2 *}$} & \multirow[b]{2}{*}{$E_{2}^{2 *}$} \\
\hline & $E_{0}$ & $E_{1}^{1}$ & $E_{1}^{2}$ & $E_{2}^{0 *}$ & $E_{2}^{0 * *}$ & $E_{2}^{1 *}$ & & & \\
\hline 1) $\lambda_{2}^{1}<\lambda_{2}^{2}<S_{2 i n}<S_{2 i n}^{* *}<S_{2 i n}^{*}$ & S & S & I & S & I & S & I & I & I \\
\hline 2) $\lambda_{2}^{1}<S_{2 i n}<S_{2 i n}^{* *}<S_{2 i n}^{*}<\lambda_{2}^{2}$ & I & $\mathrm{S}$ & & I & I & S & I & & \\
\hline 3) $\lambda_{2}^{1}<S_{2 i n}<S_{2 i n}^{* *}<\lambda_{2}^{2}<S_{2 i n}^{*}$ & I & S & & $\mathrm{S}$ & I & S & I & I & \\
\hline 4) $\lambda_{2}^{1}<S_{2 i n}<\lambda_{2}^{2}<S_{2 i n}^{* *}<S_{2 i n}^{*}$ & I & S & & $\mathrm{S}$ & I & $\mathrm{S}$ & I & I & I \\
\hline 5) $S_{2 i n}<S_{2 i n}^{* *}<\lambda_{2}^{1}<\lambda_{2}^{2}<S_{2 i n}^{*}$ & $\mathrm{~s}$ & & & $\mathrm{~s}$ & I & $\mathrm{s}$ & & I & \\
\hline 6) $S_{2 i n}<\lambda_{2}^{1}<S_{2 i n}^{* *}<\lambda_{2}^{2}<S_{2 i n}^{*}$ & S & & & S & I & $\mathrm{S}$ & I & I & \\
\hline 7) $S_{2 i n}<\lambda_{2}^{1}<\lambda_{2}^{2}<S_{2 i n}^{* *}<S_{2 i n}^{*}$ & S & & & S & I & $\mathrm{S}$ & I & I & I \\
\hline 8) $S_{2 i n}<S_{2 i n}^{* *}<\lambda_{2}^{1}<S_{2 i n}^{*}<\lambda_{2}^{2}$ & $\mathrm{~S}$ & & & I & I & $\mathrm{S}$ & & & \\
\hline 9) $S_{2 i n}<\lambda_{2}^{1}<S_{2 i n}^{* *}<S_{2 i n}^{*}<\lambda_{2}^{2}$ & S & & & I & I & $\mathrm{S}$ & I & & \\
\hline 10) $S_{2 i n}<S_{2 i n}^{* *}<S_{2 i n}^{*}<\lambda_{2}^{1}<\lambda_{2}^{2}$ & $\mathrm{~s}$ & & & $\mathrm{~s}$ & I & & & & \\
\hline
\end{tabular}

\section{Simulations numériques}

Les simulations numériques illustrées dans les Figs. 4-7 sont obtenues pour les fonctions de Monod suivantes

$$
\mu_{0}\left(X_{0}\right)=\frac{2.5 X_{0}}{1.5+X_{0}} \quad \text { et } \quad \mu_{1}(S)=\frac{2 S}{1.5+S}
$$

On choisit les valeurs suivantes des paramètres $X_{0 i n}=3, D=1, \alpha=0.75, k_{0}=1$ et $k_{1}=1.2$. On illustre le cas de bistabilité pour $\bar{S}_{1 \text { in }}=0.338<S_{1 \text { in }}=0.5<\lambda_{1}=0.9$ et tel que

$$
\xi^{\prime}\left(\frac{X_{0 i n}}{\alpha}\right)=-0.412>-0.833=-\frac{k_{0}}{k_{1}} .
$$

Dans ce cas, l'équation $\xi\left(X_{0}\right)=\delta\left(X_{0}\right)$ admet deux solutions (voir Fig. 4 à gauche) et les points d'équilibre sont donnés par

$$
F_{0}=(4,0.5,0), \quad F_{1}^{*}=(1.201,0.9,1.887) \quad \text { et } \quad F_{1}^{* *}=(2.808,0.9,0.548) .
$$

La Fig. 4 au milieu montre la convergence vers l'équilibre strictement positif $F_{1}^{*}$ pour la condition initiale $X_{0}(0)=4.5, S_{1}(0)=2$ et $X_{1}(0)=0.368$.

La Fig. 4 à droite montre la convergence vers l'équilibre de lessivage pour la condition initiale $X_{0}(0)=4.5, S_{1}(0)=2$ et $X_{1}(0)=0.367$.

La Fig. 5 met en évidence la bistabilité dans l'espace $\left(X_{0}, S_{1}, X_{1}\right)$ de dimension trois avec deux bassins d'attraction qui sont séparés par la variété stable de l'équilibre instable $F^{* *}$. Pour toute condition initiale strictement positive, la solution converge soit vers l'équilibre strictement positif $F^{*}$ soit vers l'équilibre de lessivage $F_{0}$.

La Fig. 6 à gauche illustre le cas $S_{1 i n}=1.5>\lambda_{1}$ où l'équation $\xi\left(X_{0}\right)=\delta\left(X_{0}\right)$ 


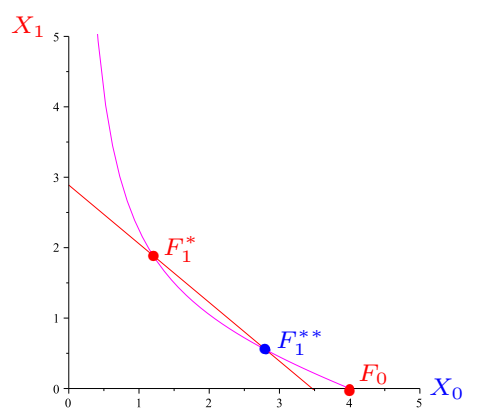

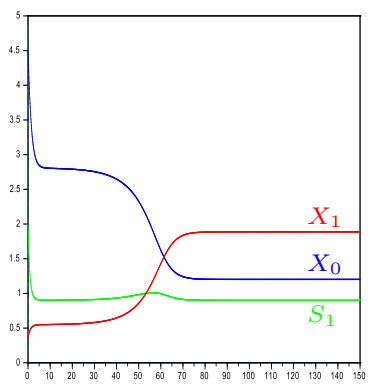

Temps

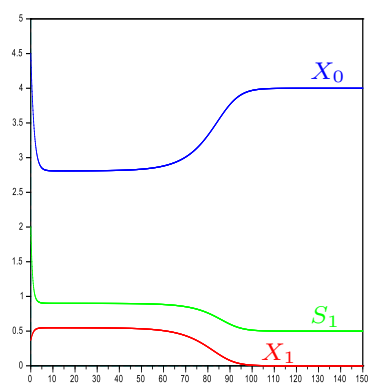

Temps

Figure 4. Le cas $0<\bar{S}_{1 \text { in }}<S_{1 i n}<\lambda_{1}$ : Existence de deux équilibres strictement positifs et bistabilité.

admet une unique solution. Les points d'équilibre sont donnés par $F_{0}=(4,1.5,0)$ et $F^{*}=(0.616,0.9,3.486)$. La Fig. 6 à droite montre la convergence globale vers l'équilibre strictement positif $F^{*}$. pour toute condition initiale strictement positive.

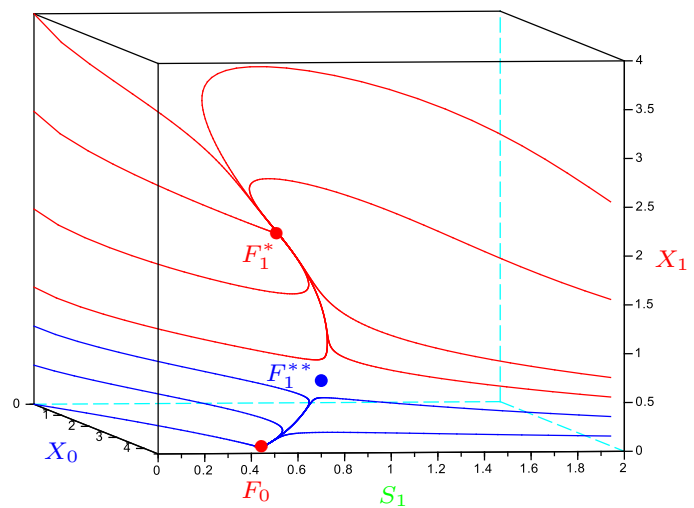

Figure 5. Bistabilité en dimension trois.

Dans la suite, on se propose d'illustrer les résultats mathématiques trouvés de l'étude du modèle à trois étapes (8). On considère les mêmes valeurs des paramètres du sousmodèle (3) et on choisit la fonction de Haldane suivante

$$
\mu_{2}\left(S_{2}\right)=\frac{m_{2} S_{2}}{K_{s}+S_{2}+\frac{S_{2}^{2}}{K_{i}}}
$$

avec $m_{2}=3, K_{s}=1$ et $K_{i}=1$. On fixe les valeurs suivantes des paramètres $S_{2 i n}=3$, $k_{2}=0.2$ et $k_{3}=1.2$. Les valeurs des seuils de rentabilité de la deuxième espèce sont données par $\lambda_{2}^{1}=0.381$ et $\lambda_{2}^{2}=2.618$. La Fig. 7 illustre le cas

$$
\lambda_{2}^{1}<\lambda_{2}^{2}<S_{2 i n}<S_{2 i n}^{* *}=3.082<S_{2 i n}^{*}=3.283
$$

avec existence de quatre points d'équilibre LAS dont un est un équilibre strictement positif où les deux espèces coexistent. La Fig. 7 (a) montre la coexistence entre les deux espèces pour une condition initiale

$$
X_{0}(0)=4.5, \quad S_{1}(0)=2, \quad X_{1}(0)=0.368, \quad S_{2}(0)=0.5, \quad X_{2}(0)=0.5
$$



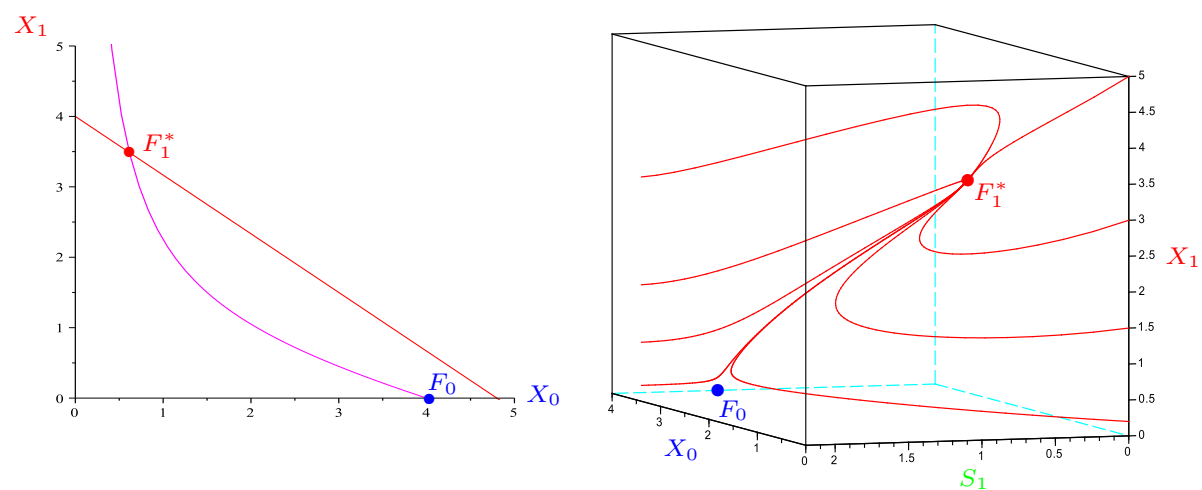

Figure 6. Le cas $S_{1 i n}>\lambda_{1}$ : Existence d'un unique équilibre strictement positif $F^{*}$ qui est GAS.

où la solution du modèle à trois étapes (8) converge vers le point d'équilibre de coexistence $E_{2}^{1 *}=(1.201,0.9,1.887,0.381,3.223)$ qui est LAS. La Fig. 7 (b) montre l'extinction de deux espèces pour une condition initiale

$$
X_{0}(0)=2, \quad S_{1}(0)=0.7, \quad X_{1}(0)=0.6, \quad S_{2}(0)=4.5, \quad X_{2}(0)=0.4
$$

et la convergence vers l'équilibre de lessivage $E_{0}=(4,0.5,0,3,0)$ qui est LAS. La Fig. 7 (c) montre le lessivage de la première espèce pour une condition initiale

$$
X_{0}(0)=1, \quad S_{1}(0)=3.5, \quad X_{1}(0)=0.2, \quad S_{2}(0)=2, \quad X_{2}(0)=2
$$

et la convergence vers l'équilibre de lessivage de $X_{1}, E_{1}^{1}=(4,0.5,0,0.381,2.908)$ qui est LAS. La Fig. 7 (d) montre l'exclusion de la deuxième espèce pour une condition initiale

$$
X_{0}(0)=2.5, \quad S_{1}(0)=0.7, \quad X_{1}(0)=1.5, \quad S_{2}(0)=4.5, \quad X_{2}(0)=0.5
$$

et la convergence vers l'équilibre de lessivage de $X_{2}, E_{2}^{0 *}=(1.201,0.9,1.887,3.283,0)$ qui est LAS.

\section{Conclusion}

Dans ce travail, nous avons étudié un modèle du chémostat avec dégradation enzymatique du substrat dont une partie peut être sous forme particulaire. Nous avons démontré que le sous-modèle, avec une modélisation particulière de l'hydrolyse qui fait intervenir un taux de croissance monotone et des concentrations du substrat à l'entrée du chémostat inférieures au seuil de rentabilité, peut présenter une bistabilité qui ne peut pas se produire dans le modèle classique du chémostat où le lessivage est l'unique point d'équilibre qui attire toutes les solutions, [8]. Cette bistabilité peut aussi avoir lieu pour une fonction de croissance non monotone, par exemple du type Haldane. On démontre ainsi, pour la modélisation de l'hydrolyse proposée, l'importance de considérer cette étape dans l'apparition des équilibres strictement positifs et de la bistabilité. Finalement, nous avons étudié le modèle à trois étapes et établi les conditions d'existence et le comportement 


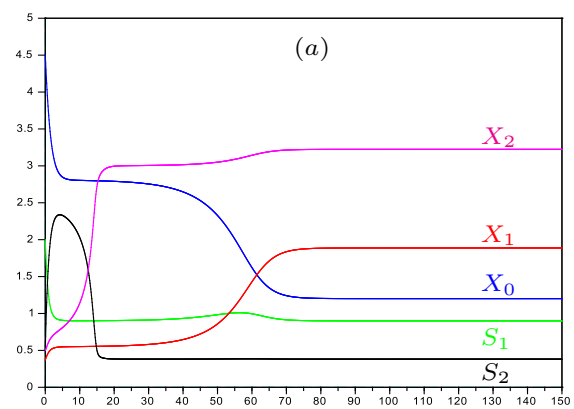

Temps

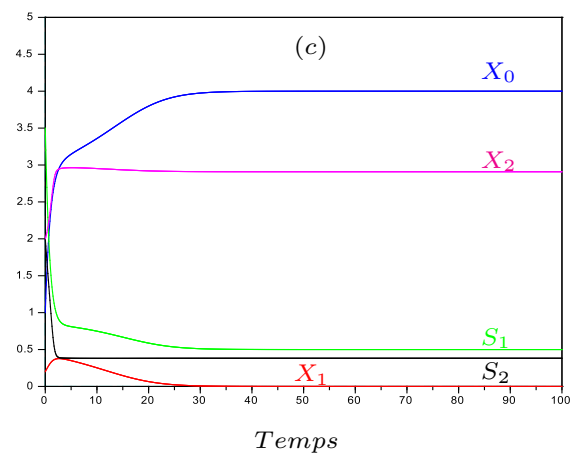

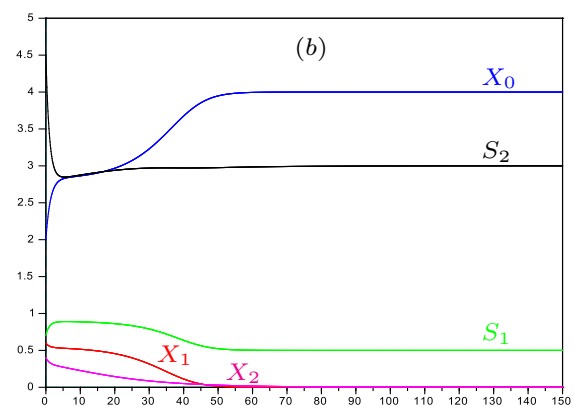

Temps

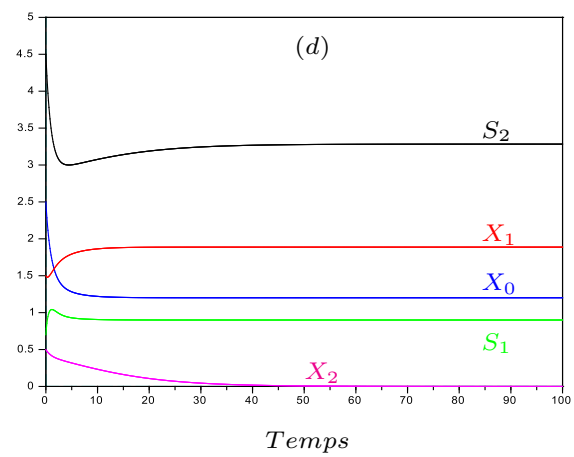

Figure 7. Le système présente une quadri-stabilité : selon la condition initiale du modèle (8), il y a soit (a) coexistence des deux espèces (convergence vers $E_{2}^{1 *}$ ), (b) lessivage des deux espèces (convergence vers $E_{0}$ ), (c) lessivage de la première espèce (convergence vers $E_{1}^{1}$ ), (d) lessivage de la deuxième espèce (convergence vers $E_{2}^{0 *}$ ).

asymptotique local des points d'équilibre. On démontre l'existence d'au plus quatre équilibres strictement positifs. L'originalité de ce travail réside dans le fait que, par rapport aux études disponibles actuellement, nous avons mis en évidence cette quadri-stabilité qui permet, en ne considérant que des conditions initiales différentes, d'obtenir des comportements qualitatifs très différents (lessivage d'une, de deux ou d'aucune des espèces). Les simulations numériques illustrent les résultats mathématiques démontrés.

\section{Remerciements}

Les auteurs remercient, en dehors de leurs institutions, le projet Euromed 3+3 TREA$\mathrm{SURE}^{1}$ et le programme Averroès ${ }^{2}$ qui ont financé leurs recherches.

1. http ://project.inria.fr/treasure/

2. http ://www.averroes.fr/averroes4/index.php/fr/

A R I M A 


\section{Bibliographie}

[1] G. BAStin, D. Dochain, « On-line estimation and adaptive control of bioreactors, Dynamics of Microbial Competition », Elsevier Science Publishers, 1991.

[2] B. BENYAhia, T.SARI, B.Cherki, J. HARMAND, « Bifurcation and stability analysis of a two step model for monitoring anaerobic digestion processes », Journal of Process Control, vol. 22, 2012, 1008-1019.

[3] B. Benyahia, T.SARI, B.Cherki, J. HARMAND, « Sur le modèle AM2 de digestion anaérobie », CARI'10, Proceedings of the 10th African Conference on Research in Computer Science and Applied Mathematics, INRIA, E. Badouel, A. Sbihi and I. Lokpo (Eds.), 2010, 453-460.

[4] O. Bernard, Z. Hadj-Sadok, D. Dochain, A. Genovesi, J. P. Steyer, « Dynamical model development and parameter identification for an anaerobic wastewater treatment process », Biotechnology and Bioengineering, vol. 75, 2001, 424-438.

[5] R. Fekih-Salem, N. Abdellatif, T. Sari, J. Harmand, « On a Three Step Model of Anaerobic Digestion Including the Hydrolysis of Particulate Matter », Proceedings of the 7th Vienna International Conference on Mathematical Modelling, 2012, 196.

[6] C. LOBRY, J. HARMAND, «A new hypothesis to explain the coexistence of N species in the presence of a single resource », CRAS série Biologie, vol. 329, 2006, 40-46.

[7] I. Simeonov, S. Stoyanov, «Modelling and dynamic compensator control of the anaerobic digestion of organic wastes », Chem. Biochem. Eng. Q, vol. 17, n 4, 2003, 285-292.

[8] H.L. Smith, P. Waltman, « The Theory of the Chemostat, Dynamics of Microbial Competition », Cambridge University Press, 1995. 